\title{
Effect of Early versus Delayed Parenteral Nutrition on the Health Outcomes of Critically Ill Adults: A Systematic Review
}

\author{
Suresh Kumar Sharma*, Ritu Rani, Kalpana Thakur \\ All India Institute of Medical Sciences- Rishikesh, Rishikesh, India
}

\begin{abstract}
Objectives: This systematic review aims to evaluate and summarise the findings of all relevant studies which identified the effect of early vs delayed parenteral nutrition (PN), early PN vs early supplemental PN and early PN vs standard care for critically ill adults. Methods: The literature search was undertaken using PubMed, Embase, Medline, Clinical Key, and Ovid discovery databases. The reference lists of studies published from 2000 till June 2020 were hand searched. Result: On screening 2088 articles, a total of five RCTs with 6,277 patients were included in this review. Only one clinical trial compared early PN and late PN; the results reported significantly shorter periods in intensive care unit $(I C U)$ stay $(p=0.02)$ and less ICU related infections $(p=0.008)$ in the late PN group compared to the Early PN group. Two trials compared total parenteral nutrition (TPN) and enteral nutrition (EN) +TPN groups. Both found a significantly longer hospital stay duration $(p<0.05$ and $p<0.01)$ with a higher mortality rate in the TPN group compared to the EN+TPN group. A statistically significant improvement was observed in patients' quality of life receiving early PN compared to standard care $(p=0.01)$. In contrast, no significant difference was found in the supplemental PN vs the standard care group. Conclusion: The supplemental PN patients had shorter ICU stay and lower mortality rates than TPN. However, these findings should be interpreted carefully as included studies have different initiation timing of nutritional support, and the patients' diagnosis varied.
\end{abstract}

Systematic review registration: PROSPERO: CRD42020183175

Keywords: early parenteral nutrition, delayed parenteral nutrition

Received: 11 October 2020 / Accepted: 11 March 2021

\section{INTRODUCTION}

Nutritional support is considered an integral element in the treatment of seriously ill patients admitted to the ICU. Optimum nutrition levels are essential to maintain competent immune function, support anabolism, reduce the metabolic response to stress, decrease oxidative cell damage, and ultimately to improve critically ill patients' health status [1]. Nutrients supplied for therapeutic reasons, whether orally, enterally, or parenterally are specified as specialised nutrition support (SNS). PN is used for patients who cannot be fed an appropriately oral diet or if the digestive tract is not functioning or inaccessible [2]. Enteral nutrition alone provides only $45 \%$ to $60 \%$ of energy due to difficulties with its provision rate. A supplemental PN strategy aims to address EN's energy gap to meet $100 \%$ of the daily energy demands. However, reports on its impact on clinical outcomes have been inconsistent in reported trials
$[3,4]$. It has been recommended that energy provision be increased from $70 \%$ to $100 \%$ of estimated needs for three to seven days of ICU post-admission $[4,5]$.

Timely initiation of optimum nutritional support is vital to limit the catabolic process and reduce adverse outcomes such as prolonged mechanical ventilation, more prolonged ICU stays, and increased mortality risk [6].

Nutritional support through the parenteral route has traditionally been associated with more significant risks and complications such as infection compared to the enteral route. However, recent developments in the supply, formulation, and monitoring of PN suggest that more up to date comparison studies and analysis of these nutritional support routes, especially in the early phase of the disease, are now required [7]. Furthermore, the use and optimal timing for PN initiation remain less well defined, also European and North American guidelines differ significantly [8]. 
The Society of Critical Care Medicine (SCCM) and the American Society for Parenteral and Enteral Nutrition (ASPEN) guidelines recommend that patients with severe malnutrition be given $\mathrm{PN}$ as soon as possible after admission to an ICU when EN is not feasible. However, in patients at low nutrition risk (NRS-2002 $\leq$ 3 or NUTRIC score $\leq 5$ ), exclusive PN is withheld over the first seven days following ICU admission [5].

Contrary wise, the 2019 European Society for Parenteral and Enteral Nutrition (ESPEN) guidelines stated that clinical nutrition should be considered for any patient staying in an ICU for more than 48 hours, and PN should be initiated within three to seven days, postadmission, when EN is contraindicated [9]. According to the Canadian clinical practice guidelines (2015), PN should not be started simultaneously with enteral nutrition and should not be used routinely in patients with an intact gastrointestinal tract. However, few data relating to patients who do not tolerate adequate enteral nutrition recommend the optimum time to initiate parenteral nutrition [10]. Moreover, there are no Indian guidelines and protocols available as to when parenteral feeding should be initiated.

Several reported studies showed the superiority of enteral nutrition over parenteral nutrition in some aspects and vice versa [11-13]. However, very few systematic reviews were explicitly conducted to explore the impact of early versus late parenteral nutrition and parenteral nutrition versus supplementary parenteral nutrition in adults. To date, no meta-analysis has been published on this issue. Also, guidelines and research results contradict parenteral nutrition, which creates more confusion regarding parenteral nutrition optimally.

This review aims to evaluate and summarise the findings of all relevant studies that identified the effect of early versus delayed parenteral nutrition, early $\mathrm{PN}$ versus early supplemental $\mathrm{PN}$, and early parenteral nutrition versus standard care in critically ill adults.

\section{MethODS}

\section{Search strategy}

This systematic review was carried out following a predetermined, written protocol registered in the PROSPERO database, registration number CRD42018092966. A broad search strategy was used to obtain all relevant studies that reported the use of early or delayed paren- teral nutrition alone or with enteral nutrition, as defined in the study protocol (Supplementary, File 1).

With a librarian's assistance, five databases, including Medline, PubMed, Embase, Ovid, and Clinical Key, were searched, and reference lists of other studies, systematic reviews, and guidelines were hand searched. A comprehensive search strategy was developed based on keywords such as "early parenteral nutrition", delayed parenteral nutrition" "supplemental parenteral nutrition", "standard care", "mortality rates", "infection rates" "length of hospital and ICU stay", "quality of life", "metabolic and gastrointestinal complications", "critically ill adults". Controlled vocabulary strategies such as Medical Subject Headings (MeSH), Emtree, and truncations were also used during the search.

Articles from January 2000 to June 2020 published in the English language were searched. Only clinical trials included patients aged 18 years or older, who had been admitted to an ICU or a postoperative unit and received parenteral nutrition alone, with or without enteral nutrition, independent of the number of calories or amount of protein intake, were included in the review. Studies, which reported any variety of composition of PN and route of PN administration, were accepted. Excluded were all clinical trials that provided enteral or oral nutrition alone in any of the comparing groups.

The experimental group was the early parenteral nutrition group. The control groups were late parenteral nutrition, supplemental parenteral nutrition, or standard care group (providing PN) as per the existing hospital protocol). The outcomes for which data had sought were categorised as primary outcomes such as ICU mortality, length of ICU and hospital stay, quality of life, and secondary outcomes such as infection, organ failure, metabolic and gastrointestinal complications. All the statistics reported in the review were derived from the searched articles.

\section{Selection of studies and data extraction}

Two reviewers independently screened the titles and abstracts for potentially relevant studies. The full text of all eligible studies was retrieved and reviewed by the same reviewers for inclusion in the review. Agreements between the two reviewers for the inclusion of studies were assessed. Disagreements were settled by consensus and discussion with the third reviewer.

The relevant articles' searching and the selection process is presented as PRISMA (Preferred Report- 
ing Items for Systematic Review and Meta-Analysis) flow chart (Figure 1). A total of five RCTs with a total of 6,277 patients were included in this review. Two authors independently extracted the selected studies' data, including essential characteristics of the studies (Table 1) and relevant primary and secondary outcomes of the included studies (Table 2).

\section{Assessment of risk bias in included studies}

Two authors independently assessed the risk of bias of the included studies using the Cochrane 'Risk of Bias' tool [14]. The risk of bias was assessed for each of the domains as low, high, or unclear; any disagreement was settled by discussion, and if not resolved, then the third author was consulted.

Selection bias (random sequence generation), attrition bias, reporting bias, and other biases were not identified in any of the five included studies [15-19]. However, two trials $[16,17]$ had an unclear risk of bias in some domains, such as selection bias (allocation concealment), performance bias, and detection bias. A high risk of bias was found in four studies, of which three studies $[15,18,19]$ had a high risk of performance bias and detection bias. One study [17] had a high risk of allocation concealment selection bias. The 'Risk of
Bias' assessments for each included study in graphs and figures is summarised in Figures 2 and 3. PRISMA guidelines [20] were followed throughout the preparation of this review.

\section{Assessment of heterogeneity and data synthesis}

The initial plan was to conduct a systematic review and meta-analysis to integrate the findings of potentially relevant clinical trials to find the best possible solution of initiation timings of the (supplemental) parenteral nutrition in critically ill adults. In addition to the unavailability of the potentially relevant clinical trials, there were inconsistencies in the interventions, basic characteristics of the patients, and the concept of early and late parenteral nutrition among the included studies that did not allow us to conduct a meta-analysis. Therefore, a systematic review was carried out. Furthermore, an agreement of the definition of "early PN" and "late PN" was problematic due to the presence of heterogeneity. Clinical and methodological heterogeneity was analysed through an informal analysis of study characteristics using clinical judgement [14,21,22].

Hence, this systematic review was carried out with narrative syntheses of the studies' results.
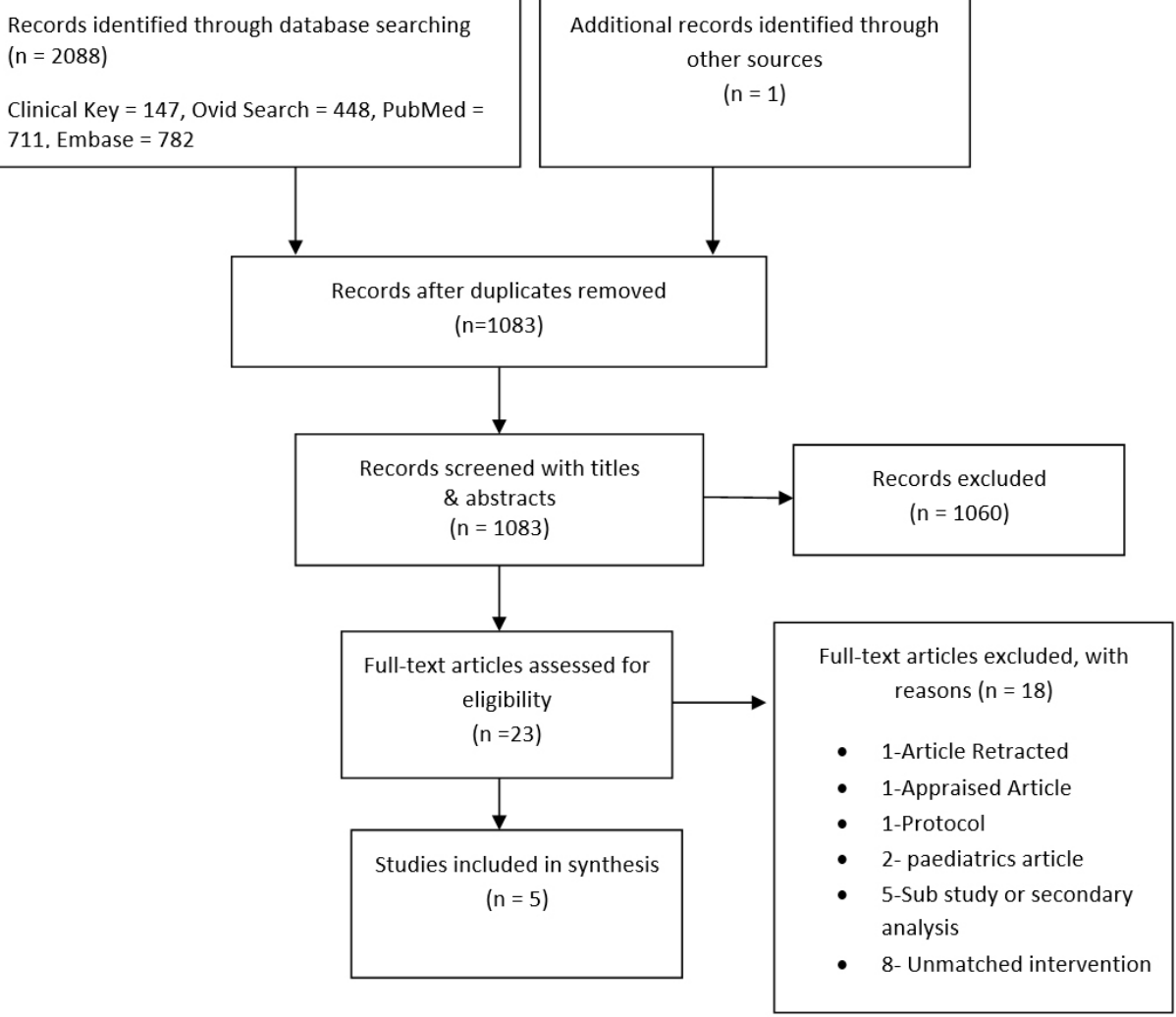

Fig. 1. PRISMA flow chart of search strategy and selection of articles 


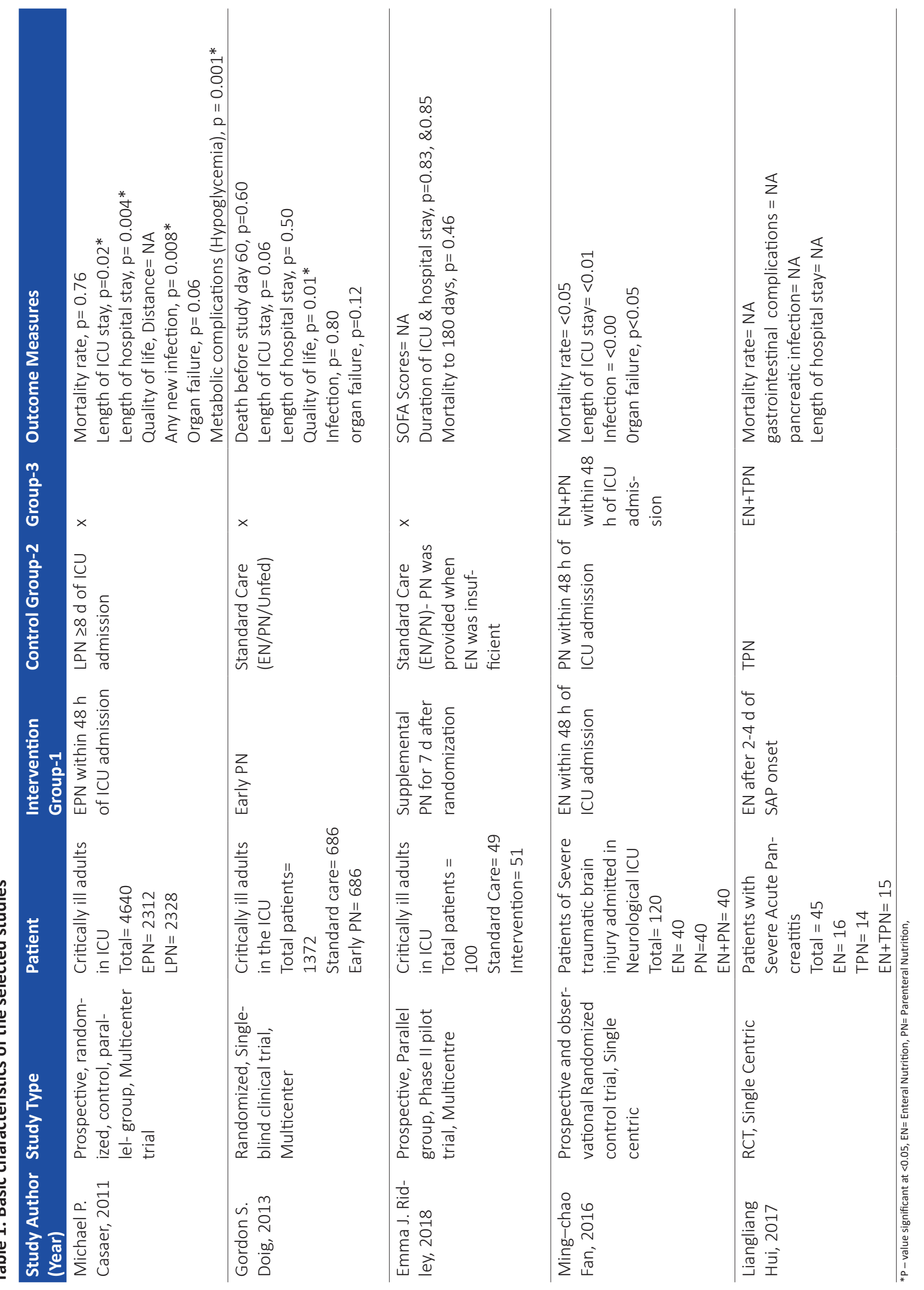




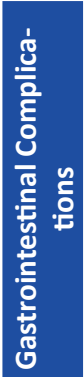

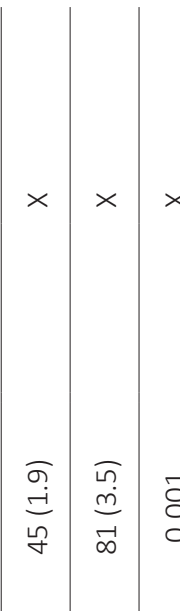
를 崖

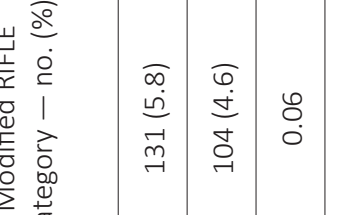

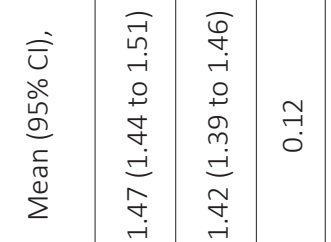

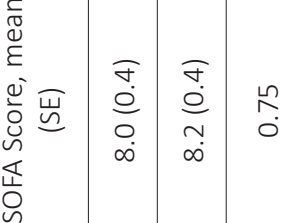

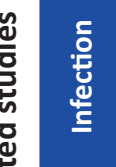
总

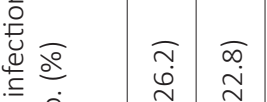
음

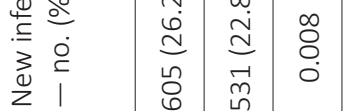

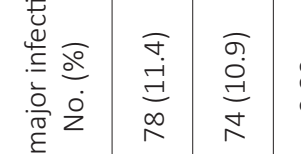

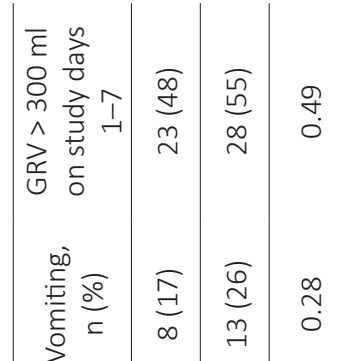

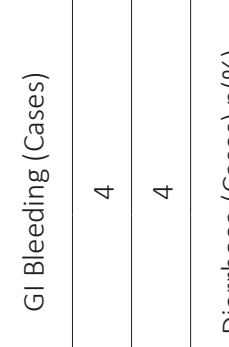

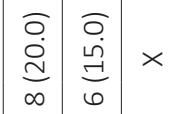 安 定

冚 


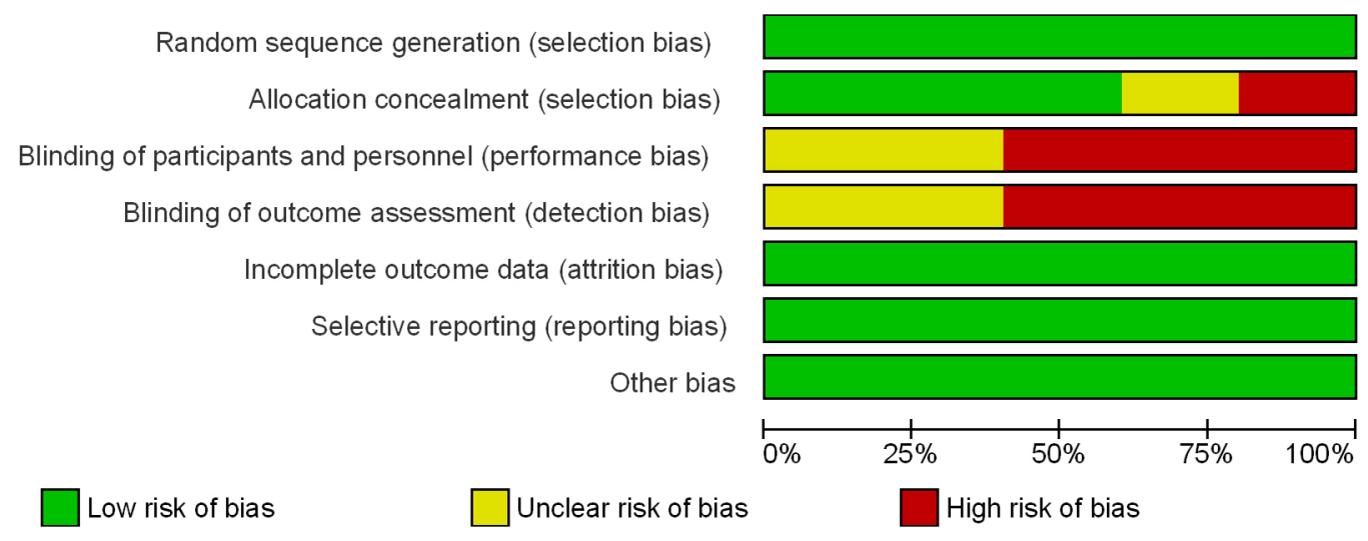

Fig. 2. Risk of bias graph: review authors' judgements about each risk of bias item presented as percentages across all included studies.

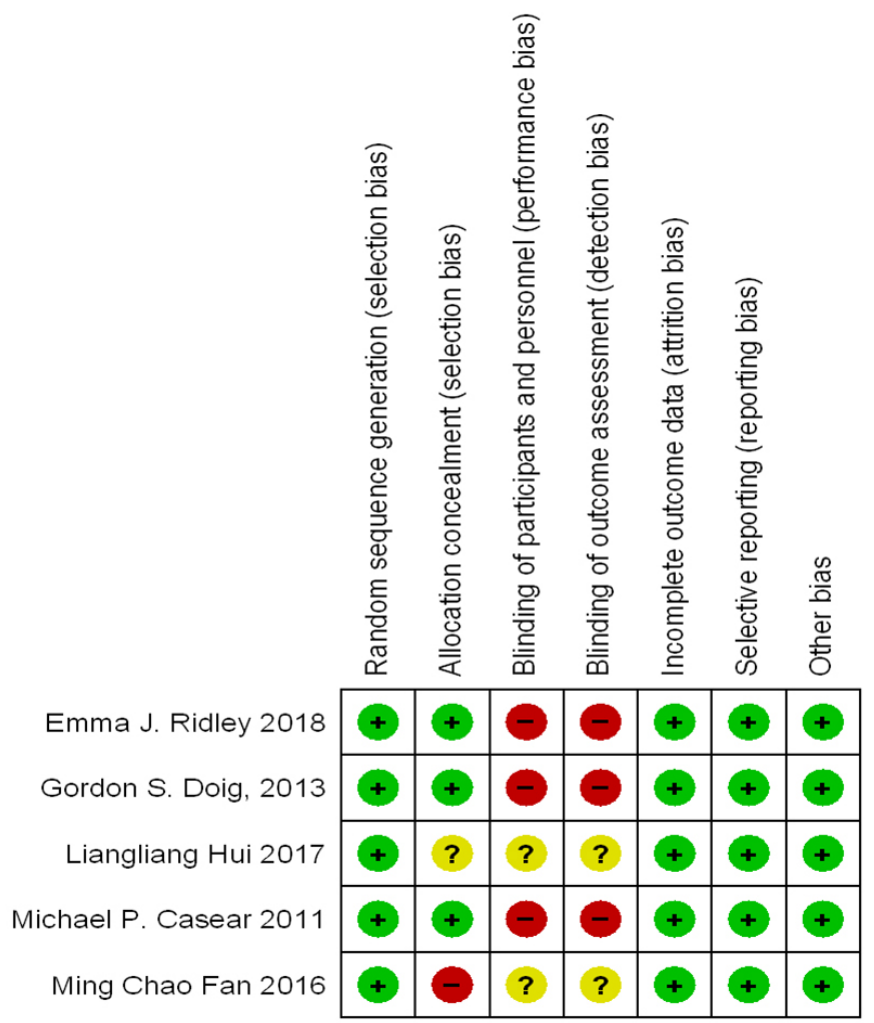

Fig. 3. Risk of bias summary: review authors' judgements about each risk of bias item for each included study

\section{RESULTS}

A total of 2088 potentially relevant articles were identified via searching Embase ( $\mathrm{N}=782)$, PubMed $(\mathrm{N}=711)$, Ovid Search $(\mathrm{N}=448)$, Clinical Key $(\mathrm{N}=147)$. Additional records were included through other sources $(n=1)$. After removing duplicates, 1083 articles were screened based on their titles and abstracts. Twenty-three fulltext articles were reviewed based on the inclusion and exclusion criteria of the review. Of these, eighteen articles were excluded as they did not meet the eligibility criteria. One retracted trial was identified, one ap- praised article, two paediatric trials, one ongoing trial, five were sub-study or secondary analysis of a major clinical trial (EPaNIC study), and eight studies had an unmatched intervention. Five RCTs were finally identified for inclusion in this systematic review (Figure 1).

The review included five trials that enrolled 6,277 participants, ranging from 45 to 4640 participants. The mean age of the participants ranged from 41.6 and 68.6 years in all included trials. Three of the five reported studies $[15,18,19]$ related to critically ill medical and surgical adult patients. One of the studies [17] had en- 
rolled participants with traumatic brain injury, and one trial [16] had patients with severe acute pancreatitis. Of the total clinical trials included, one trial [15] compared EPN and LPN, one trial compared early TPN and standard care [18], one trial [19] compared supplemental PN with standard care, and two trials $[16,17]$ compared TPN and supplemental PN. Basic characteristics and clinical outcomes of the included studies are given in Table 1 and 2, respectively.

\section{Length of ICU and hospital Stay}

Caesar M. (2011) [15] found a shorter period of ICU stay in the late-initiation PN group ( $\geq 8 \mathrm{~d}$ of ICU admission) compared with the early initiation PN group, i.e., within $48 \mathrm{~h}$ of ICU admission. For more than three days, more patients in the EPN group remained in the ICU than the patients in the LPN group [15]. Compared to the EN+TPN group, the TPN group had a slightly longer median hospital stay during discharge [16,21]. Fan M. (2016) [17] also reported that the mean ICU length of stay was more in the PN group as compared to the $\mathrm{EN}+\mathrm{PN}$ group. Hence, it is interpreted that supplemental PN had a shorter length of ICU and hospital stay compared to PN alone. Compared to the standard treatment, there was no substantial difference in the duration of the ICU and hospital stay compared to the early TPN group [18]. Ridley E. (2018) [19] also did not report any significant difference in median days of ICU and hospital stay of supplemental PN versus standard care.

\section{Mortality}

Caesar M. (2011) [15] reported similar mortality rates in EPN and LPN groups at the ICU and the hospital. However, in the LPN group, the probability of earlier discharges alive from the ICU and the hospital increased by $6.3 \%$ without reducing functional status. However, more patients developed hypoglycaemia in LPN compared to the EPN group [15]. In the PN group, mortality rates were significantly greater than in the EN+TPN group [17]. Similarly, Hui L. (2019) [16] reported more cases in the PN group than EN+TPN. Hence, it is considered that supplemental parenteral nutrition is superior to parenteral nutrition alone in terms of mortality rate. Whereas, in contrast with standard treatment versus early $\mathrm{PN}$, there was no significant difference in study day-60 mortality, death before ICU and hospital discharge [18]. In another study, Ridley E. (2018) [19] also did not find any significant difference in the number of 90 days and 180 days' survival of patients in the usual care and EPN group.

\section{Quality of life}

Functional status was similar in the two study groups (EPN and LPN), as measured by the six-minute walking distance and daily living activities at the time of incipient hospital discharge [15]. A statistical improvement in patients' quality of life receiving early parenteral nutrition is more likely than those receiving standard-care as measured using RAND-36 General Health Status. However, the difference's size did not surpass half the standard deviation and was not considered clinically significant [18]. According to Ridley E. (2018)[19], there was no significant difference in the quality of life of usual care versus supplemental PN group as measured using EuroQuol-5 Dimension 3 Level (EQ-5D-3L) at hospital discharge, 90 and 180 days after enrolment.

\section{Other complications - Infection}

Patients in the late-initiation group had lesser ICU infections than the early-initiation group and lower cholestasis incidence; fewer patients in the late initiation group had new infections in the airways or lungs, bloodstream, wound, and urinary tract. However, the acute inflammatory reaction was more marked in the early PN group, as evident with the median C-reactive protein level [15]. Comparing the PN group with the $\mathrm{EN}+\mathrm{PN}$ group showed that complication rates were significantly lower for intracranial infection and pyemia in the PN group. [17]. However, there were no significant differences between groups (standard care vs early TPN) in new infection rates [18].

\section{Organ Failure}

There was a median reduction of three days in the renal replacement therapy of late than the early parenteral nutrition group [15]. Doig G (2013) reported no significant reduction in organ failures in early TPN versus standard care groups [18].

No significant difference was found in the usual care versus intervention group's Sequential Organ Failure Assessment scores [19].

\section{Metabolic and gastrointestinal complications}

More number of patients in the late $\mathrm{PN}$ group had high bilirubin levels (> $3 \mathrm{mg} / \mathrm{dl}$ ), and fewer had a clinically significant rise in GGT (gamma-glutamyl transferase) 
or ALP (alkaline phosphatase) levels. The number of patients with an increase in aminotransferase levels was comparable in both groups (LPN and EPN), while hypoglycaemia was more profound in the LPN group than the EPN group [15]. The TPN and EN+TPN groups showed significantly increased MODS cases or death at discharge, prolonged hospital stays, and increased cases of gastrointestinal bleeding and pancreatic infection compared to the EN group. However, in the TPN and EN+TPN groups, cases of abdominal distension, regurgitation, or aspiration were significantly reduced compared to the EN group [16]. Ridley E. (2018) [19] did not report any substantial reduction in the number of patients in the usual care versus intervention group with gastric residual volume exceeding $300 \mathrm{ml}$, abdominal distension and vomiting.

\section{Discussion}

The review was divided into three objectives to understand better the initiation timings of the supplemental parenteral nutrition. The primary outcomes were mortality, quality of life, length of ICU, and hospital stay. The secondary outcomes were complications such as infection, organ failure, and metabolic and gastrointestinal complications.

Caesar M. (2011) [15] reported similarities in early (within 48 hours) and late parenteral nutrition group (after seven days) mortality rates, but also a $6.3 \%$ rise in the likelihood of earlier ICU and hospital alive discharges in the late parenteral nutrition group. In support, Shah S. (2019) [21] also reported no significant differences in mortality rates between early (within seven days) and late (after seven days) PN groups. However, it was also evident in other studies that early PN reduces patients' hospital stay $[18,21]$. On comparing early $\mathrm{PN}$ with the supplemental $\mathrm{PN}$ group, the RCTs evidenced higher mortality rates in the early PN group than the supplemental PN group [16,17]. However, a large multicentre observational study suggested that the provision of protein and calories to critically ill patients could improve with supplemental parenteral nutrition; no clinical benefit was identified [22].

Moreover, clinical trials involving standard care versus supplementary PN showed no significant variations in mortality rates in both groups $[18,19]$. Thus, patients in the early or late parenteral nutrition group, supplementary PN group, and the standard treatment group were assumed to have no significant differences in mortality rates. However, the standard treatment in both trials was different $[18,19]$. Doig G (2013) included nearly $40 \%$ of patients who eventually received delayed enteral nutrition; some patients received delayed enteral or parenteral nutrition. Some remained unfed during their ICU stay [18]. However, Ridley E. (2018) [19] described the standard treatment as offering PN when EN was inadequate. At the same time, the intervention group provided a supplemental PN approach administered for up to seven days, and the total average energy and protein requirement were different in both the standard treatment group and the supplemental PN group, i.e., 2092 (392) kcal, and 103 (21) g respectively.

A shorter ICU stay and the probability of earlier discharge of the patients from hospital care was observed in the late initiation PN group, when treatment was delayed for more than eight days after ICU admission, compared to early initiation of treatment. Patients in the EPN group remained in the ICU for three days more than the LPN group patients [15]. In contrast, Shah S. (2019) [21] reported a significantly shorter hospital stay in the EPN group (within seven days) than the LPN group (after seven days) of admission.

However, when PN was supplemented with EN, an ICU stay was significantly prolonged in the PN group compared to the SPN group [16,17]. It has been found that there was no difference between the functional status, as assessed by a six-minute walk, and in daily living activities, of EPN and LPN group at the time of hospital discharge [15]. In contrast, it has been reported that early parenteral nutrition was found to be protective against both muscle wasting and fat loss, with significant early benefits appearing to persist over the patient's entire ICU stay compared to standard treatment [18]. Correspondingly, a study found that patients who had undergone PN post-surgically for gastric cancer can substantially improve the quality of life, nutritional and psychological status, and patients' immune functions [23].

In terms of infection, it was found that fewer patients acquired new infections in their respiratory tracts, blood circulation, or wounds. However, there were a more pronounced acute inflammatory response and hyperbilirubinemia in the LPN group than in the early initiation group. Cases with a rise in aminotransferase levels were similar in both the LPN and EPN group [15]. After doing sub-analysis of the study done by Casaer M. (2011) [15], the EPaNIC trial of early PN versus late $\mathrm{PN}$ found that tolerating significant caloric 
deficit by restricting PN until day eight of critical illness increased plasma bilirubin but decreased the incidence of biliary sludge and decreased gamma-glutamyl transferase, alkaline phosphatase and alanine aminotransferase (GGT, ALP) [24]. A secondary analysis of the EPaNIC trial showed that postponing any PN use to supplement insufficient EN beyond one week in the ICU significantly reduced the risk of acquiring an invasive fungal infection in the ICU by $32 \%$ [25]. Similarly, it has been observed that feeding patients to cover an individualised measured energy target with supplemental PN from day four was associated with improved immunity, less systematic inflammation, and less muscle mass loss. A lower intracranial infection and pyemia were also found in the supplemental PN group than the PN group [17].

When the TPN and supplemental parenteral nutrition were compared, both the groups had a higher number of multiple organ dysfunction syndromes, increased cases of gastrointestinal bleeding. However, cases of abdominal distention, regurgitation, or aspiration were significantly lower than the EN group [16]. In the same way, no significant difference was observed in the rates of new infections, incidences of organ failures, and cases having gastric residual volume more than $300 \mathrm{ml}$, abdominal distension, and vomiting between the standard care groups and early TPN $[18,19]$. These discrepancies between the researches regarding the health outcomes of the patients receiving parenteral nutrition could be due to the heterogeneity in the study sample, i.e. patients with medical illness, surgical procedures, neuro-trauma [17], and severe acute pancreatitis [16]. So, the energy requirement of the patients varies accordingly. This is in agreement with a systematic review that emphasised that differences between the groups for nutritional delivery and energy requirements cannot be ignored as a possible factor for nosocomial infection. However, it is not known how, irrespective of the composition of nutritional support, an increased number of calories may affect nosocomial infection [26].

It is said that though PN is more straightforward to deliver than EN, its metabolic consequences are more difficult to manage [27]. Metabolic PN status such as blood glucose, normal urea, creatinine, and electrolytes such as chloride, bicarbonate, phosphate, plasma triglycerides, magnesium, and signs of infection should be routinely assessed.
A recent report on current practice and standards in the United Kingdom stated that, while delivering PN, "good practices" were followed in only $19 \%$ of the cases. Furthermore, there was a reported lack of supervision and assessment in $54 \%$ of managed patients. Also, $\mathrm{PN}$-related metabolic complications occurred in $40 \%$ of cases, out of which $49 \%$ of these complications could have been prevented if necessary steps had been taken [28].

The monitoring issue is usually not discussed in nutrition recommendations, even though it is crucial in achieving success with any therapy [29]. For critically ill patients, using a straightforward approach means opposing one nutritional approach over others is no more a fair solution. An early aggressive feeding strategy, delivering full estimated energy targets from day one, was inappropriate regardless of the route [26].

\section{- CONCLUSION}

This review found that patients on supplemental PN had shorter ICU stays and lower mortality rates than those on PN alone; however, these results should be interpreted carefully as included studies have different patients' conditions, varying nutritional support composition and intervention timings. Hospitals should have fixed feeding protocols regarding early over delayed parenteral nutrition and early $\mathrm{PN}$ over standard treatment. This would ensure better feeding and nutritional status among hospitalised patients.

\section{CONFLICT OF INTEREST}

None to declare.

\section{DEFERENCES}

1. Marik P. Enteral Nutrition in the Critically III: Myths and Misconceptions. Critical Care Medicine. 2014; 42(4):962-969.

2. Guidelines for the use of parenteral and enteral nutrition in adult and pediatric patients [Internet]. PubMed. 2021 [cited 7 March 2021]. Available from : https://pubmed.ncbi.nlm.nih. gov/11841046/

3. Ridley E, Davies A, Parke R, Bailey M, McArthur C, Gillanders L et al. Supplemental parenteral nutrition in critically ill patients: a study protocol for a phase II randomised controlled trial. Trials. 2015;16(1).

4. Yamamoto S, Allen K, Jones K, Cohen S, Reyes K, Huhmann M. Meeting Calorie and Protein Needs in the Critical Care Unit: A Prospective Observational Pilot Study. Nutrition and 
Available online at: www.jccm.ro

Metabolic Insights. 2020;13:117863882090599.

5. Taylor B, McClave S, Martindale R, Warren M, Johnson $D$, Braunschweig $C$ et al. Guidelines for the Provision and Assessment of Nutrition Support Therapy in the Adult Critically III Patient: Society of Critical Care Medicine (SCCM) and American Society for Parenteral and Enteral Nutrition (ASPEN). Critical Care Medicine. 2016;44 (2):390-438.

6. Kapoor M, Ramprasad R. Nutrition in intensive care. Journal of Anaesthesiology Clinical Pharmacology. 2012;28(1):1.

7. Harvey S, Parrott F, Harrison D, Sadique M, Grieve R, Canter R et al. A multicentre, randomised controlled trial comparing the clinical effectiveness and cost-effectiveness of early nutritional support the parenteral versus the enteral route in critically ill patients (CALORIES). Health Technology Assessment. 2016;20 (28):1-144

8. Kerrie J, Bagshaw S, Brindley P. Early versus late parenteral nutrition in the adult ICU: feeding the patient or our conscience? Canadian Journal of Anesthesia/Journal canadiend'anesthésie. 2012;59(5):494-498.

9. Gostyńska A, Stawny M, Dettlaff K, Jelińska A. Clinical Nutrition of Critically III Patients in the Context of the Latest ESPEN Guidelines. Medicine. 2019;55(12):770.

10. 2015 - Critical Care Nutrition [Internet]. Criticalcarenutrition. com. 2021 [cited 7 March 2021]. Available from: https://criticalcarenutrition.com/resources/cpgs/pastguidelines/2015.

11. Pacelli F. Enteral vs Parenteral Nutrition After Major Abdominal Surgery. Archives of Surgery. 2001;136(8):933.

12. Harvey S, Parrott F, Harrison D, Bear D, Segaran E, Beale R et al. Trial of the Route of Early Nutritional Support in Critically III Adults. New England Journal of Medicine. 2014;371(18):16731684.

13. Simpson F, Doig G. Parenteral vs enteral nutrition in the critically ill patient: a meta-analysis of trials using the intention to treat principle. Intensive Care Medicine. 2004;31(1):12-23.

14. Cochrane Handbook for Systematic Reviews of Interventions [Internet]. Handbook-5-1.cochrane.org. 2021 [cited 7 March 2021]. Available from: https://handbook-5-1.cochrane.org/

15. Casaer M, Mesotten D, Hermans G, Wouters P, Schetz M, Meyfroidt $G$ et al. Early versus Late Parenteral Nutrition in Critically III Adults. New England Journal of Medicine. 2011;365 (6):506-517.

16. Hui L, Zang K, Wang M, Shang F, Zhang G. Comparison of the Preference of Nutritional Support for Patients With Severe Acute Pancreatitis. Gastroenterology Nursing. 2019;42(5):411416.
The Journal of Critical Care Medicine 2021;7(3) • 169

17. Fan $\mathrm{M}$, Wang $\mathrm{Q}$, Fang $\mathrm{W}$, Jiang $\mathrm{Y}, \mathrm{Li}$ L, Sun $\mathrm{P}$ et al. Early Enteral Combined with Parenteral Nutrition Treatment for Severe Traumatic Brain Injury: Effects on Immune Function, Nutritional Status and Outcomes. Chinese Medical Sciences Journal. 2016;31(4):213-220.

18. Doig G. Early Parenteral Nutrition in Critically III Patients With Short-term Relative Contraindications to Early Enteral Nutrition. JAMA. 2013;309(20):2130.

19. Ridley E, Davies A, Parke R, Bailey M, McArthur C, Gillanders $L$ et al. Supplemental parenteral nutrition versus usual care in critically ill adults: a pilot randomised controlled study. Critical Care. 2018;22(1).

20. PRISMA [Internet]. Prisma-statement.org. 2021 [cited 7 March 2021]. Available from: http://www.prisma-statement. org/PRISMAStatement/Checklist

21. Shah S, Hollands J, Pontiggia L, Bingham A. Impact of the Time to Initiation of Parenteral Nutrition on Patient Outcomes in Critically III Adults. Nutrition and Metabolic Insights. 2019;12:117863881985931.

22. Kutsogiannis J, Alberda C, Gramlich L, Cahill N, Wang M, Day A et al. Early use of supplemental parenteral nutrition in critically ill patients: Results of an international multicenter observational study*. Critical Care Medicine. 2011;39(12):2691-2699.

23. Jin Y, Yong C, Ren K, Li D, Yuan H. Effects of Post-Surgical Parenteral Nutrition on Patients with Gastric Cancer. Cellular Physiology and Biochemistry. 2018;49(4):1320-1328.

24. Vanwijngaerden $Y$, Langouche $L$, Brunner $R$, Debaveye $Y$, Gielen $\mathrm{M}$, Casaer $\mathrm{M}$ et al. Withholding parenteral nutrition during critical illness increases plasma bilirubin but lowers the incidence of biliary sludge. Hepatology. 2014;60(1):202-210.

25. De Vlieger G, Ingels C, Wouters P, Debaveye $\mathrm{Y}$, Vanhorebeek I, Wauters J et al. Impact of supplemental parenteral nutrition early during critical illness on invasive fungal infections: a secondary analysis of the EPaNIC randomised controlled trial. Clinical Microbiology and Infection. 2019;25(3):359-364.

26. Wang D, Lai X, Liu C, Xiong Y, Zhang X. Optimal Timing for the Initiation of Supplemental Parenteral Nutrition in Critical Conditions- A Mini-Review. Quality in Primary Care.2015; 23 (6): 333-335

27. Berger M, Pichard C. Parenteral nutrition in the ICU: Lessons learned over the past few years. Nutrition. 2019; 59:188-194.

28. Macdonald K, Page K, Brown L, Bryden D. Parenteral nutrition in critical care. Continuing Education in Anaesthesia Critical Care \& Pain. 2013;13(1):1-5.

29. Singer $\mathrm{P}$, Blaser $\mathrm{A}$, Berger $\mathrm{M}$, Alhazzani $\mathrm{W}$, Calder $\mathrm{P}$, Casaer $\mathrm{M}$ et al. ESPEN guideline on clinical nutrition in the intensive care unit. Clinical Nutrition. 2019;38(1):48-79. 